\title{
Demand Side Load Management for Big Industrial Energy Users under Blockchain-Based Peer-to-Peer Electricity Market
}

\author{
Can Dang ${ }^{1}$, Jiangfeng Zhang ${ }^{2 *}$, Chung-Ping Kwong ${ }^{3}$, and $\mathrm{Li} \mathrm{Li}^{2}$
}

\begin{abstract}
Blockchain is the key technology of Bitcoin and other cryptocurrencies, and it is one of the most exciting technologies changing the world in the 2010's. Targeting at big industrial energy users, this paper first presents a new market structure (i.e., transaction rules) under existing blockchain-based electricity transaction platforms to cover popular types of markets such as contract, day-ahead, adjustment and balancing markets, and then focuses on the optimal load management problem for a particular industrial user. The proof-of-work cost from blockchain is also modelled. A key feature of this load management problem is that the user has direct control on its own load, and the obtained load control model is much more accurate than existing approaches in which system operators or demand aggregators cannot control load directly and have to rely on inaccurate estimations. As a case study, the pumping load of a water supply plant is investigated to illustrate how the demand load is managed under this blockchainbased market. From the case study, it is found that $18.9 \%$ of total cost can be saved under this new market structure.
\end{abstract}

Index Terms-Blockchain, Demand side management, Peer-topeer energy market, Electricity market.

Nomenclature
subscript $w$
subscript $t$
subscript $b$
superscript $B$
superscript $S M$
superscript $D A$
superscript $A$
$P_{i}(t)$
$\lambda, p(t)$
$\Delta^{+}, \Delta^{-}$
$E_{b, t, w}^{B}, E_{t, w}^{D A}, E_{t, w}^{A}$
$r_{t, w}^{+}, r_{t, w}^{-}$
$r$
$\alpha$
$\beta$

index for uncertainty scenario

index for time

index for contract type

index indicating bilateral contract

index indicating spot market

index indicating day-ahead market

index indicating adjustment market

power consumption of the $i$-th appliance electricity price

positive or negative amount of imbalance

energy in the relevant markets

ratio between positive (or negative)

imbalance price and DA price

proof-of-work unit price

probability in $\mathrm{CVaR}$

weighting factor on risk and cost

\section{INTRODUCTION}

*: Corresponding author;

${ }^{1}$ State Key Laboratory of Electrical Insulation and Power Equipment, School of Electrical Engineering, Xi' an Jiaotong University, 710049, China, can.dang_cn@yahoo.com;

${ }^{2}$ School of Electrical and Data Engineering, University of Technology Sydney, NSW 2007, Australia, \{jiangfeng.zhang, li.li\}@uts.edu.au;

${ }^{3}$ C.P. Kwong Consultant and the Chinese University of Hong Kong, Hong Kong, cpkwong@cpkwong.com
D ISTRIBUTED blockchain technology appeared in October 2008 in Ref. [1] under an alias of Satoshi Nakamoto to support the first cryptocurrency Bitcoin, and the Bitcoin network was started in January 2009. Since then, Bitcoin gradually entered into the finance industry, and becomes the most influential cryptocurrency. The blockchain technology behind Bitcoin turns to be a game-changing innovation for the whole world, and there are many industries that will be disrupted by blockchain, such as legal industry, financial services, life sciences, health care, cyber security, supply chain management, private transport and ride sharing, cloud storage, charity, voting, government, public benefits, energy management, retail, and real estate amongst others [2], [3]. The applications of blockchain in distributed energy generation [4], renewable and carbon credits [5], and data security of power systems against cyber-attacks [6] are also very promising. Therefore, existing electricity market needs to be reformed to take advantage of this new technology, and this paper intends to study how a big industrial user can best manage its load under such a new blockchain-based electricity market.

In practice, there are already many pilot projects applying blockchain technology in electricity market at small regional levels. For example, LO3 Energy has run a peer-to-peer (P2P) energy market under their TransActive Grid private blockchain protocol at the Brooklyn microgrid in New York [7], and the Australian government has granted AU\$8 million in 2017 to apply Power Ledger's blockchain technology for distributed energy and water systems at the City of Fremantle, where Ethereum blockchain, POWR tokens and Smart Bond contracts are key tools in the Power Ledger Platform ${ }^{1}$. Cofunded by the Australian Renewable Energy Agency, the LO3 Energy is also leading a blockchain-based virtual microgrid project at Latrobe Valley in Victoria, Australia, in $2018^{2}$. A comprehensive review is provided in [8] on the basic principles of blockchain, 140 blockchain-based energy trading projects, and the relevant technical challenges and market barriers. It is interesting to note that blockchain-based P2P energy market is a special form of general P2P energy market, while a more general concept is transactive energy [9] which can cover any existing P2P energy trading mechanisms.

Blockchain-based P2P energy trading research has been carried out along with these commercial pilot projects. Seven

\footnotetext{
${ }^{1}$ https://www.powerledger.io

${ }^{2} \mathrm{https} / / /$ arena.gov.au/news/latrobe-valley-virtual-microgrid-allow-dairyfarms-trade-energy-via-blockchain/
} 
components of a blockchain-based microgrid energy market are proposed in [7], and a blockchain protocol based on smart contracts is applied to build a high-performing information system. Blockchain is also employed in [10] to establish a machine-to-machine electricity market in the context of chemical industry, and energy transactions are recorded and validated by a private blockchain-based software system MultiChain. A two-layer energy trading system based on multi-agent and blockchain is proposed in [11] to facilitate P2P market. For electric vehicles, a consortium blockchain for local aggregators is proposed in [12] to audit and validate electricity trading among plug-in hybrid electric vehicles, and a new energy blockchain is proposed in [13] to enable electric vehicles to publicly audit and share transactions without reliance on any trusted intermediary. The consortium blockchain approach in [12] is also extended to general energy blockchain transactions for the purpose of credit-based payment and transaction security [14]. A token-based decentralised energy trading system using blockchain, multi-signatures and anonymous encrypted messaging streams is discussed in [15] to solve challenges from the security and privacy of consumption and trading data.

Within the blockchain-based P2P electricity market, it is very challenging to optimise the benefit of a particular electricity buyer under complicated market operations and demand side management (DSM) strategies. There are many helpful studies on optimal energy trading decision makings in P2P, or more generally, transactive energy markets, although the markets discussed are not blockchain-based. For example, price competition among sellers is modelled as an evolutionary game, and interactions between sellers and buyers is modelled as a Stackelberg game in [16] to study the $\mathrm{P} 2 \mathrm{P}$ trading within a community. A residential DSM optimisation model is proposed in [17] to minimise the total household energy costs under P2P. Benefits of flexible load and energy storage are also demonstrated in a 4-layer P2P trading system in [18]. Real-time and forward markets are introduced for bilateral contracts in P2P energy trading in [19]. The role of energy brokers is studied through reinforcement learning for indirect customer-to-customer energy trading in [20]. A specific P2P trading system for electric vehicles is proposed in [21] to reduce the impact of charging process on power systems during business hours. Various game- and auction-theoretic approaches in P2P energy trading are discussed in [22], [23], and in particular, Nash bargaining theory is applied to develop a bilateral transactive energy trading framework in [24] for multiple participants. Physical low-voltage network constraints are considered using sensitivity analysis in [25] for P2P trading. Power losses are allocated for microgrid P2P blockchain market participants in [26]. At the power distribution level, reference [27] proposes a day-ahead transactive energy market which helps the distribution system operators to optimally decide the schedules of distributed energy resources, while [28] introduces a new P2P energy market based on the concept of multi-class energy management to coordinate trading among prosumers with heterogenous preferences.

From the above literature study, it is noted that existing studies have made significant contributions to establish the P2P electricity transaction platform by blockchain technology; however, the cost minimisation problem for any big industrial user has not been investigated yet; market structure integrating bilateral contract, day-ahead (DA), adjustment and balancing markets is not studied; and market participants often do not have the authority to control the load directly, and thus the relevant load has to be approximated by inaccurate models, such as an approximated range of flexible load [16]. A big industrial energy user is a very special entity in the $\mathrm{P} 2 \mathrm{P}$ electricity market. It often has sufficient resources to organise its own P2P energy market, and it can also participate in any existing $\mathrm{P} 2 \mathrm{P}$ market. No matter which kind of market it will organise or participate, this big user has the authority to directly control its own load, and therefore, it has a great opportunity to reduce its electricity cost by exercising load management techniques under any given P2P market. It is worth noting that blockchain technology provides a general transaction platform and we can build further new market structures upon existing blockchain $\mathrm{P} 2 \mathrm{P}$ market platforms to reduce transaction cost and increase energy supply security. Under the existing blockchain energy trading platforms (e.g., [7]) and technologies such as information collection, data communication, and transaction ledgers, this paper further proposes a new market structure (i.e., transaction rules) for big industrial energy users to organise their own P2P market, which is a combination of contract, DA, adjustment and real-time balancing markets, and then the load management strategies of such a big energy user are optimised under this P2P market to save its electricity cost. Stochastic programming approach for electricity market pool from [29] is adopted here to model the overall operational cost and the risk of higher cost under market uncertainties. That is, high cost risks caused by market uncertainties are mitigated by minimising the conditional value-at-risk (CVaR) calculated from possible scenarios. A key feature of this paper is that load can be directly controlled by the big user for cost minimisation purpose, and the results will be much more accurate than existing studies on demand aggregators who do not have the authority for direct load control and the responded demand has to be modelled by inaccurate approximations.

The remaining part of this paper is organised as follows. The P2P market structure for a big industrial consumer is designed in Section II. The corresponding DSM strategies for the industrial user under this P2P market are modelled as a stochastic programming problem in Section III. A case study for the DSM of a water supply plant under P2P market is presented in Section IV. Finally, conclusions are drawn in Section V.

\section{DEMAND SIDE P2P MARKET DESIGN FOR A BIG INDUSTRIAL CONSUMER}

A big industrial energy consumer can either participate in any existing electricity market, or organise its own P2P market. Now consider the case that this consumer will organise its own blockchain-based P2P market using technologies from any existing P2P energy trading platforms. The electricity suppliers can be traditional power generation plants or small scale renewable generations from prosumers. Current electricity 
purchase and electricity market structure [29] are followed for this consumer, that is, the consumer can either buy electricity through bilateral contracts, or through organising an electricity pool market such as a DA market, an adjustment market (i.e. intra-day market), and a real-time balancing market. A combination of both bilateral contract and the self-organised electricity pool market is also possible and thus considered here in this paper. In the following, the basic structures of these trading methods are briefed.

i) Bilateral contract: A big consumer can sign bilateral contracts with suppliers for a fixed time period. For example, a contract can be signed for each week/month/year, and the contracted electricity price can be determined by the supplier's available tariff choices, or the average of a contracted reference price and the main grid spot market price (see, for example, Chapter 9 of [29]). The consumer can also sign DA contracts with the suppliers for the amount of energy purchased in the next day, since this DA contract will enable the consumer to forecast more accurately its demand of the next day.

ii) Electricity pool market: The big consumer can organise the following three types of electricity pool markets simultaneously, or keep only the DA and the balancing markets. It can also organise only the DA market. Such decisions on market organisation depend on the needs and available resources of the consumer.

- DA market: In the DA market, the consumer estimates its demand for the next 24 hours, offers its demand in the form of 24 blocks of hourly energy consumption $\left(P^{i}\right)$ and the corresponding price that the customer is willing to pay $\left(p^{i}\right), i=1,2, \cdots, 24$. The suppliers bid the corresponding generations and the corresponding prices for this 24-hour period. The demand data and supplier offers must be submitted before a fixed time, e.g. 10:00am, on the day before the day of actual power delivery.

- Adjustment market: The adjustment market allows the consumer and suppliers to modify their offers from the clearing time of the DA market to a few hours before the actual consumption, and it can be cleared every few hours.

- Balancing market: In the real-time balancing market, imbalance between the supply and demand from the DA market is compensated 15 minutes or any other fixed time interval before each delivery hour. Similar to the dual price-balancing method in [30], the market clearing prices for positive imbalance and the negative imbalance may be different.

While the consumer is organising these markets, the actual transactions need to satisfy the relevant security constraints as required by the distribution system operator or transmission system operator. This kind of security constraints along with the relevant network congestion issues are solved by nodal or zonal pricing mechanism in existing electricity markets. In this $\mathrm{P} 2 \mathrm{P}$ market, a similar security-constrained bid-based generation dispatch can be determined following a similar approach.

In the traditional market, the big consumer can buy electricity directly from the grid or electricity market pool at contracted or spot market price. This consumer can also be a prosumer which has self-power generation facilities, e.g., photovoltaic (PV) or wind, to supply its own load or trade with other consumers or generations; see, for example, the market sharing model for a P2P PV prosumer in [31]. In this paper, we assume that the self-generated power is much less than the load since this is a big industrial user. Therefore, the self-generated power will not be sold to other users through the P2P market.

With the above market structure, power transactions can be realised through any existing blockchain energy market platform. That is, existing blockchain electricity market technologies, such as the ledgers to record transactions, and the information processing system to communicate and store data [7], [8], will be adopted to implement the proposed structure on contract, DA, adjustment and balancing markets. It is worth noting that the target of introducing the blockchainbased $\mathrm{P} 2 \mathrm{P}$ market is to reduce unnecessary transaction cost, however, the blockchain framework is not completely free to use, and there is the proof-of-work or proof-of-state cost in all the existing cryptocurrencies and other practical energy blockchain projects [8], although such a cost could be very little compared with existing charges of various brokers and agents.

\section{DSM UNDER BLOCKCHAIN P2P MARKET}

Under the proposed market structure mentioned in Section II, the objective of the demand side remains the same as before, i.e., it is still to minimise its equipment operational cost. This operational cost mainly consists of its electricity cost, and is determined by both the equipment power consumptions and the corresponding electricity tariff. Consider a particular $\mathrm{P} 2 \mathrm{P}$ electricity trade over a fixed time period $\left[t_{0}, t_{f}\right]$. This is typically a 24-hour period similar to current DA electricity market, and it can also be a trade for a shorter time duration. For simplicity, assume this time duration $\left[t_{0}, t_{f}\right]$ is the 24hour period of a day starting from 0 o'clock. In mathematical terms, the electrical cost over a fixed time period $\left[t_{0}, t_{f}\right]$ can be written as the following function

$$
f_{e}(P, p)=\int_{t_{0}}^{t_{f}} \sum_{i=1}^{N} P_{i}(t) p(t) \mathrm{d} t,
$$

where $P(t)=\left(P_{1}(t), P_{2}(t), \cdots, P_{N}(t)\right), P_{i}(t)$ is the power consumption of the $i$-th electrical appliance at time $t, p(t)$ is the electricity price at $t$, and $N$ is the total number of electrical appliances. Assume that the consumer has a bilateral contract, and also organises three types of markets; therefore, the energy price $p(t)$ will consist of prices coming from the bilateral contract and the three types of markets, and the relevant $P(t)$ will be the corresponding power consumption under these contracts or markets. In discrete form, the electricity cost can be rewritten as follows when the sampling period is taken as $\Delta t$, which equals 1 hour in most applications.

$$
\begin{aligned}
& f_{e}(P, p, b)=\sum_{w=1}^{N_{W}} \sum_{t=1}^{N_{T}} \pi_{w}\left(\lambda_{b t w}^{B} E_{b t w}^{B}+\lambda_{t, w}^{D A} E_{t, w}^{D A}\right. \\
& \left.\quad+\lambda_{t, w}^{A} E_{t}^{A}+\lambda_{t, w}^{D A} r_{t, w}^{+} \Delta_{t, w}^{+}-\lambda_{t, w}^{D A} r_{t, w}^{-} \Delta_{t, w}^{-}\right),
\end{aligned}
$$

where $N_{W}$ scenarios of possible market price are considered, $\pi_{w}$ is the occurrence probability of each scenario, $\lambda_{b t w}^{B}$ is the electricity price of type $b$ contract at time $t$ under scenario $w$ (in most of the situations only one type of contract is signed, and thus here only the type $b$ contract is considered), 
$E_{b t w}^{B}$ is the corresponding amount of energy purchased; $\lambda_{t, w}^{D A}$ and $\lambda_{t, w}^{A}$ are the electricity price of the DA and adjustment markets at time $t$ under scenario $w$, respectively; $E_{t, w}^{D A}$ and $E_{t, w}^{A}$ are the amount of electricity purchased in the DA and adjustment markets at time $t$ in scenario $w$, respectively; $\Delta_{t, w}^{+}$and $\Delta_{t, w}^{-}$are the amount of positive and negative imbalance, respectively. Notation $r_{t, w}^{+}\left(\right.$or $\left.r_{t, w}^{-}\right)$represents the ratio between the positive (or negative) imbalance market price and the corresponding DA market price. These market prices and the relevant models are standard approach widely accepted in literature (see, e.g., [29], [30], [32], [33]). The contract price $\lambda_{b t w}^{B}$ is determined by a similar approach as [29]:

$$
\lambda_{b t w}^{B}=\frac{\lambda_{b}^{B}+\lambda_{t w}^{S M}}{2}, \forall t, w
$$

where $\lambda_{b}^{B}$ is the reference contract price, $\lambda_{t w}^{S M}$ is the spot market price. The average with the spot market price will help the consumer to hedge the risk of higher spot market price.

As mentioned earlier in the end of the previous section, there will be the 'proof-of-work' or 'proof-of-state' cost in this market similar to the Bitcoin or Ethereum system which awards participants to solve crypto-problems and add new blocks to the existing blockchain. This kind of cost is usually a fixed cost for each new block added. For the electricity $\mathrm{P} 2 \mathrm{P}$ transactions, the amount of energy transacted can be exactly measured, and therefore, it is proposed to charge a very small rate for the amount of energy traded and transfer this charge to successful participants adding new blocks to the main blockchain. This proof-of-work cost can also be shared between the customer who buys energy and the suppliers who sell energy. Anyway, the customer will bear either part or full of the proof-of-work cost which is represented by the following function.

$$
f_{p w}(P)=r \sum_{w=1}^{N_{W}} \sum_{t=1}^{N_{T}} \pi_{w}\left(E_{t, w}^{D A}+E_{t}^{A}+\Delta_{t, w}^{+}-\Delta_{t, w}^{-}\right),
$$

where $r$ is a fixed small rate in $\$ / \mathrm{kWh}$ for proof-of-work cost paid by the customer, and the consumer's proof-of-work payment is proportional to the total amount of energy traded.

To prevent the consumer from intentionally ordering more or less electricity than needed, it is assumed that a constant penalty price $\lambda_{B}$ (in $\$ / \mathrm{kWh}$ ) needs to be paid by the consumer to suppliers for the total volume of imbalance $\left(\Delta_{t, w}^{+}+\Delta_{t, w}^{-}\right)$ for each scenario $w$ (see also [33]). Then the risk cost of the volume imbalance is

$$
f_{\text {penalty }}=\lambda_{B} \sum_{w=1}^{N_{W}} \sum_{t=1}^{N_{T}} \pi_{w}\left(\Delta_{t, w}^{+}+\Delta_{t, w}^{-}\right) .
$$

The above volume imbalance risk is a penalty charge that the customer must pay in case of imbalance. Now consider the high cost risk measure, which is characterised as the concept of CVaR [34]. CVaR measures the average risk of high cost, and is calculated by the following formula for any given small probability $\alpha \in(0,1)$ :

$$
C \operatorname{VaR}_{\alpha}(Z)=\inf _{t \in \mathbb{R}}\left(t+\alpha^{-1} E\left\{[Z-t]_{+}\right\}\right),
$$

where $Z=f_{e}(P, p)+f_{p w}(P)+f_{\text {penalty }}$ is the random variable representing the total cost of the customer, $E\{\cdot\}$ is the mathematical expectation, and the function $[\cdot]_{+}$is defined as $[a]_{+}=\max \{a, 0\}$ for any real number $a \in \mathbb{R}$ ([35]). Similar to [29], [34], the above $C V a R$ is equivalent to the minimum value of the following linear programming problem:

$$
\begin{array}{rc}
C V a R_{\alpha} & =\min \left(\zeta+\frac{1}{\alpha} \sum_{w=1}^{N_{W}} \pi_{w} \eta_{w}\right) \\
\text { s.t. } & f_{e}(P, p)+f_{p w}(P)+f_{\text {penalty }}-\zeta \leq \eta_{w}, \forall w \\
\eta_{w} \geq 0, \forall w .
\end{array}
$$

Therefore, the objective of the consumer is to minimise its following total cost.

$$
\begin{aligned}
\min & {\left[(1-\beta) *\left(f_{e}(P, p)+f_{p w}(P)+f_{\text {penalty }}\right)\right.} \\
& \left.+\beta *\left(\zeta+\frac{1}{\alpha} \sum_{w=1}^{N_{W}} \pi_{w} \eta_{w}\right)\right]
\end{aligned}
$$

which is subject to the following constraints:

$$
\begin{array}{r}
f_{e}(P, p)+f_{p w}(P)+f_{\text {penalty }}-\zeta \leq \eta_{w}, \forall w \\
\eta_{w} \geq 0, \forall w, \\
g(P)=0, \\
h(P) \geq 0,
\end{array}
$$

where $\beta \in[0,1]$ is a weighting factor representing the preference on cost and risk, $g(P)=0$ and $h(P) \geq 0$ represent the general load management and system constraints which can be identified whenever particular customer information is given. Note that in most of the existing studies on the offering strategies of generators (e.g. [33], [32]), the controllable load is often assumed to be freely changeable within an interval [16] due to the lack of sufficient load information and also the lack of authority to control loads. For the P2P big consumer scenario discussed here, the consumer has direct control on its load; therefore, the load management constraints (11)-(12) are available to flexibly minimise its operational cost. Detailed formulation of these constraints will be introduced by the case study in the next section.

\section{CASE STUDY FOR A WATER SUPPLY PLANT}

In this section, a water supply plant is investigated to show how the electricity cost is minimised under this P2P market. The water supply system is illustrated in Fig. 1 [36], and it has 21 pumps supplying 4 reservoirs.

The 21 pumps are divided into four groups (denoted by $N_{1}, \cdots, N_{4}$ ) depending on the destination reservoir that the pumps supply. That is, the pumps No.19, 20, 21, 14, 1, 2, 3 , and 4 from group $N_{1}$ pump water to the first destination reservoir; the pumps No. 5, 6, and 7 from group $N_{2}$ pump water to the second reservoir; the pumps No. 8, 9, 10, 11, 12 from group $N_{3}$ pump water to the third reservoir; and the pumps No. 13, 15, 16, 17, and 18 from group $N_{4}$ pump water to the fourth reservoir. The water demand of the reservoirs are independent to each other. The 21 pumps are limited to the total electricity consumption $P_{\max }$ and the total volume of incoming water per hour. 


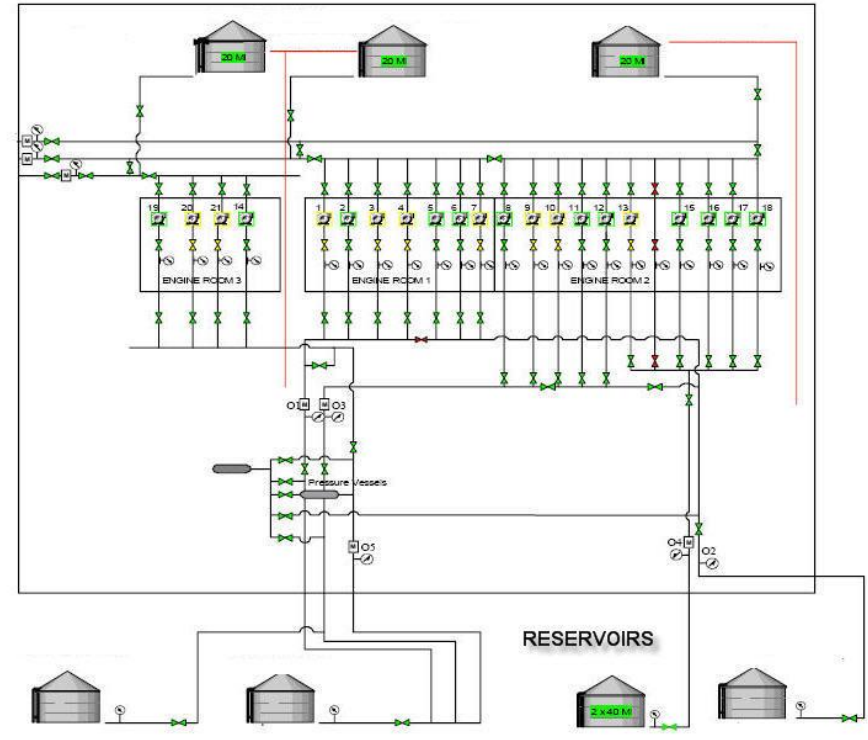

Fig. 1. Water Supply System [36]

A 24-hour control period is considered in this case study. The hydraulics of the water distribution system is modeled using the mass balance equation as formulated below ([36])

$$
R_{t, w}^{k}=\widetilde{V}_{t w}^{k}-D_{t}^{k}+R_{t-1, w}^{k},
$$

where $R_{t, w}^{k}$ is the volume of water in the $k$-th reservoir at time $t$ (i.e., the $t$-th hour), $D_{t}^{k}$ is the water demand requirement of the end user of the $k$-th reservoir at time $t$, and $\widetilde{V}_{t w}^{k}$ is the total volume of water pumped by the pumps to the $k$-th reservoir at time $t$. Without loss of generality, we can assume that the water demand $D_{t}^{k}$ does not rely on electricity price due to the lack of any time-of-use water tariff and a direct link between the water supplier's electricity cost and the water end users' water cost. Therefore, it will not change under different price scenario $w$ and there is no need to introduce any notation like $D_{t w}^{k}$.

The scheduling of pumps can be formulated as a linear programming problem when the pumps are controlled by variable speed drives. For this purpose, assume that the switching status of the $i$-th pump at the $t$-th hour under scenario $w$ is represented by $u_{i t w}$ which belongs to the interval $[0,1]$. The value 1 denotes switching on, 0 denotes switching off, while any intermediate value between 0 and 1 denotes how much percentage of the maximum power is actually being extracted. Therefore, $\left\{u_{i t w}: i=1, \cdots, N ; t=1, \cdots, 24 ; w=1, \cdots\right.$, $\left.N_{w}\right\}$ will be the variables of the minimisation problem for the cost function (8). The following constraints must be met.

1) Total amount of water pumped to each reservoir: For $k=1,2,3,4$, the sum of the volumes of water pumped by the operating pumps at the $k$-th group equals to the previously defined notation $\widetilde{V}_{t w}^{k}$ :

$$
\begin{gathered}
\sum_{i \in N_{k}} V_{i} u_{i t w}=\widetilde{V}_{t w}^{k}, t=1,2, \cdots, 24 \\
\quad k=1,2,3,4 ; w=1, \cdots, N_{w}
\end{gathered}
$$

where $V_{i}$ denotes the volume of water that the $i$-th pump can pump within one hour when the pump works at its maximum power $P_{i}$.

2) Reservoir Constraints: The volume of water in the $k$-th reservoir should be between the maximum and minimum specified levels, which are denoted by $R_{\max }^{k}$ and $R_{\min }^{k}$ respectively. For simplicity and also to include the worst case, we suppose the initial volumes of water inside the reservoirs equal to the minimal possible level $R_{\min }^{k}$. Then we have

$$
\begin{aligned}
& R_{\min }^{k} \leq R_{t}^{k} \leq R_{\max }^{k}, \\
& R_{1}^{k}=R_{\min }^{k}, t=2,3, \cdots, 24 ; k=1,2,3,4 .
\end{aligned}
$$

3) Switching Constraints: The switching function $u_{i t w}$ must satisfy

$$
0 \leq u_{i t w} \leq 1, \quad \forall i, t, w .
$$

4) Peak Demand and Security Constraints: The energy utilised by the 21 operating pumps must be less than or equal to the specified maximum electricity demand $P_{\text {max }}$.

$$
\sum_{i=1}^{21} P_{i} u_{i t w} \leq P_{\max }, 1 \leq t \leq 24 ; 1 \leq w \leq N_{w} .
$$

When the power distribution network is experiencing or will experience security problems such as thermal limit violations, significant voltage or frequency drops, then the system operator will take the relevant measures to maintain secure operations, for example, to require the big end user's total power consumption to be restricted to certain values (denoted by $P_{\text {security }}$ ) at certain peak hours. This can be simply written as the following inequality for specific time period $t$ which is often within peak hours.

$$
\sum_{i=1}^{21} P_{i} u_{i t w} \leq P_{\text {security }}, 1 \leq w \leq N_{w} .
$$

5) Volume Constraints: The volume pumped by the pumps within one hour must be less than or equal to the incoming water supply rate $V_{\text {supply }}$ (Ml/hour), where Ml denotes Megalitre, and $V_{\text {supply }}$ is a constant. Then

$$
\sum_{i=1}^{21} V_{i} u_{i t w} \leq V_{\text {supply }}, 1 \leq t \leq 24 ; 1 \leq w \leq N_{w} .
$$

6) Energy Balance:

$$
E_{b t w}^{B}+E_{t w}^{D A}+E_{t w}^{A}+\Delta_{t w}^{+}-\Delta_{t w}^{-}=\sum_{i=1}^{N} P_{i} u_{i t w} \Delta t
$$

The overall optimisation problem is to solve the operational cost minimisation problem of the water supply plant in the P2P market, restrained by the P2P market structure, blockchain technique implementation, and the inherent constraints of the water supply plant. Mathematically, the optimisation problem is to solve (8) subject to constraints (9), (10), (13)-(20). The optimisation variables are $\left\{E_{b t w}^{B}, E_{t w}^{D A}, E_{t w}^{A}, \Delta_{t w}^{+}, \Delta_{t w}^{-}, u_{i t w}\right.$, $\zeta, \eta_{w}: t=1, \cdots, 24 ; w=1, \cdots, N_{w} ; i=1, \cdots$, 


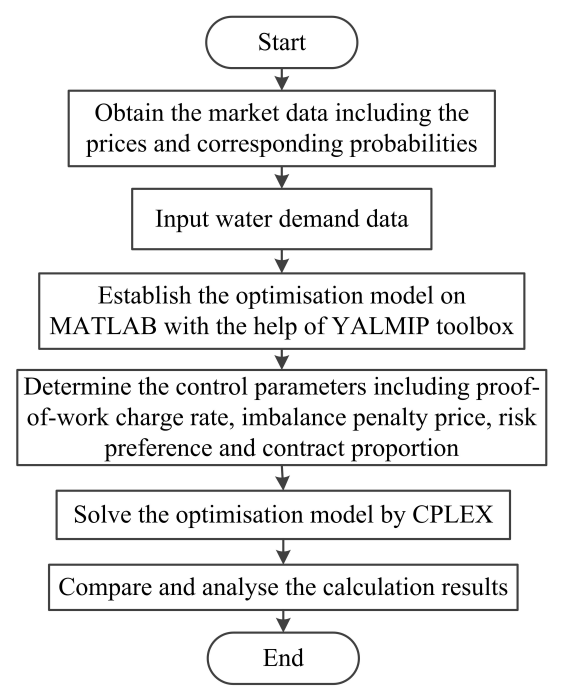

Fig. 2. Solution process

$N\}$, which are all real variables. The costs $f_{e}(P, p), f_{p w}(P)$ and $f_{\text {penalty }}$ in the objective function (8) take the form of linear weighted cost of each scenario, and the calculation of CVaR is transformed into a linear programming problem. The constraints (9), (10), (13)-(20) are all linear constraints. Therefore, the optimisation problem is a linear programming problem, and the computational burden mainly lies in the size of data. In this paper, we adopt the linear optimisation software CPLEX to solve the problem. The mathematical model is built on the platform of MATLAB, with the help of YALMIP toolbox to translate the mathematical models into programming codes. The flow chart of the solving process is illustrated in Fig 2.

Uncertainties in electricity market prices are modelled by scenarios generation results from [37], where historical data of market prices from the Iberian Peninsula electricity market are taken as example, and the uncertainties of the problem are modeled through a scenario tree with 300 scenarios $(10 \times$ $5 \times 6$ ) including 10,5 , and 6 scenarios for DA, adjustment and balancing market prices, respectively. The water supply plant data are from [36]. After studying historical electricity consumption data of the plant, only minor changes are found within any single month's period when different 24-hour load profiles are compared with each other, and therefore, monthly average 24-hour load profile is used to represent the electrical load.

\section{A. Base Case}

A base case illustrating the application of blockchain technology in P2P based electricity market is discussed in this subsection, and it is denoted as Case A for comparison purpose. The raw data of Case A are shown as follows.

The prices of the contract, DA market, adjustment market and balancing market are illustrated in Figs. 3-6.

We set the parameters $\alpha=0.07, r=0.01, \lambda_{B}=0.05$ and $\beta=0.07$. Considering that the volume of water pumped into the reservoirs will roughly equal the amount of water consumed

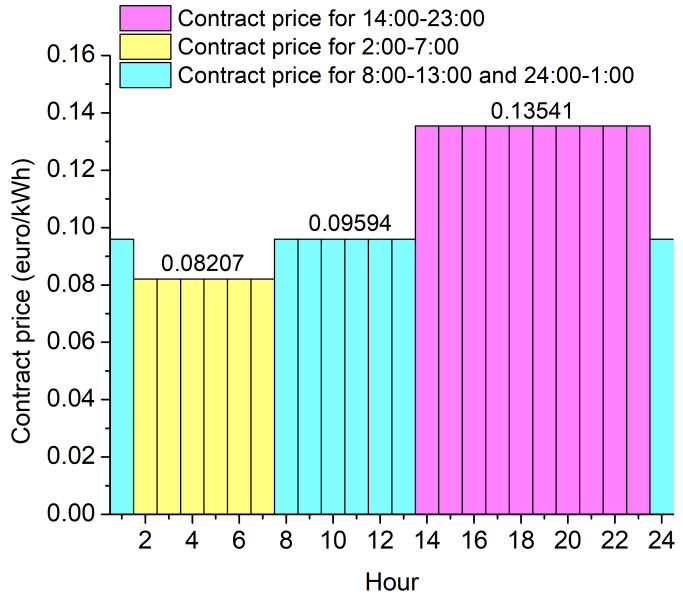

Fig. 3. Contract price

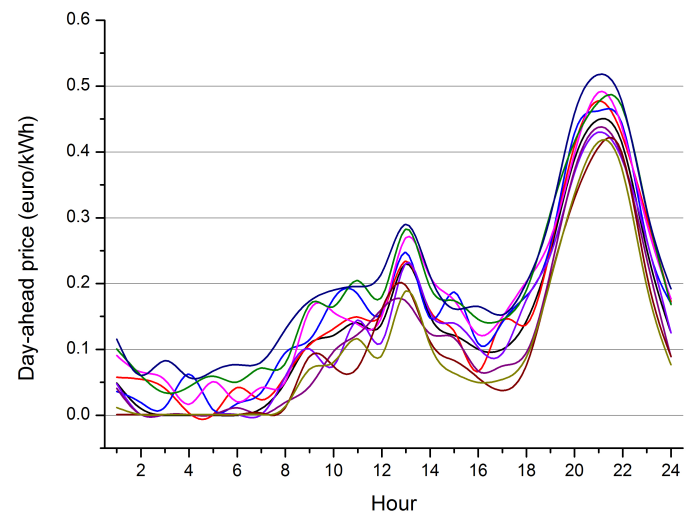

Fig. 4. DA market price

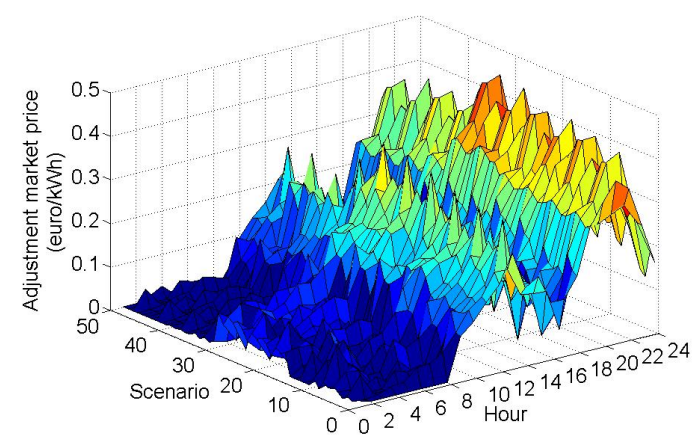

Fig. 5. Adjustment market price

over a long term period, e.g., one year, therefore, here it is assumed that the amount of water pumped into the reservoir will be greater than or equal to the amount of water users requested. Security constraint (18) is not considered in Case A since it will be discussed in Case C. The obtained optimisation result is shown in Table I. The electricity bought from the contract, DA market, adjustment market and balancing market accounts for $44.6 \%, 36.0 \%, 19.4 \%$ and $0.03 \%$ of the total 


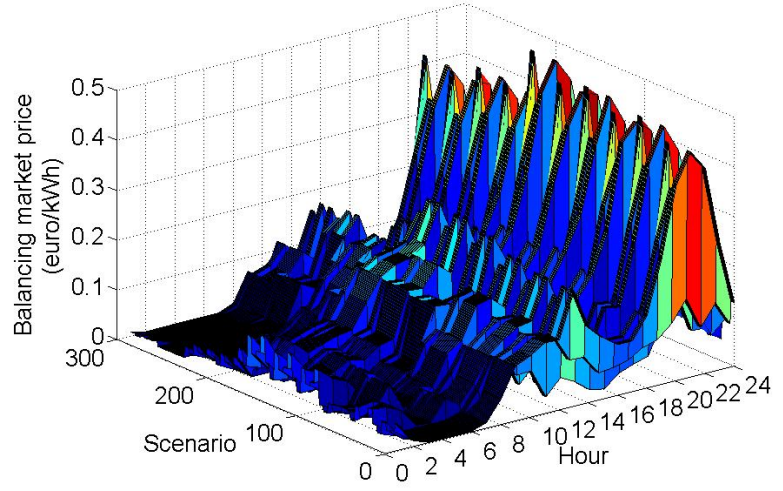

Fig. 6. Balancing market price

TABLE I

OPTIMISATION RESULTS OF THE BASE CASE

\begin{tabular}{lll}
\hline \hline Electricity bought from $(\mathrm{kWh}):$ & Contract & 306775 \\
& DA market & 247706 \\
& Adjustment market & 133210 \\
& Balancing market & 234 \\
\hline Electricity sold to $(\mathrm{kWh}):$ & Balancing market & 329 \\
\hline Cost of buying electricity from $(€):$ & Contract & 31067 \\
& DA market & 13303 \\
& Adjustment market & 6259 \\
& Balancing market & 17 \\
\hline Cost of selling electricity to $(€):$ & Balancing market & 36 \\
\hline Cost of proof-of-work $(€)$ & & 35 \\
\hline Penalty of unbalance $(€)$ & & 0.26 \\
\hline Electricity buying \& selling cost $(€)$ & & 50645 \\
\hline Cost of risk $(€)$ & & 2491 \\
\hline Final total cost $(€)$ & & 53136 \\
\hline \hline
\end{tabular}

amount, respectively. The amount of electricity bought from the balancing market is very small, and is $234 \mathrm{kWh}$ only. As a result, the costs on buying electricity from the contract, DA market, adjustment market and balancing market make up $58.5 \%, 25.0 \%, 11.8 \%$ and $0.07 \%$ of the total cost, adding up to $95.4 \%$ of the total cost, with the remaining $4.6 \%$ accounting for the cost of risk, proof-of-work, and the penalty of unbalance. In fact, the amount of electricity bought from the balancing market almost equals that of electricity sold to the balancing market, both of which are at a relatively low level. Therefore, the cost of buying electricity from the balancing market almost counteracts with the income of selling electricity to the balancing market. Within the $4.6 \%$ of total cost on risk, proof-of-work and penalty of unbalance, the cost of proof-of-work is 35 euros (€), making up only $0.07 \%$ of the total cost. The penalty of unbalance also accounts for a small proportion of $0.0005 \%$ of the total cost. As we set the factor of risk preference $\beta$ at 0.07 , the cost of risk is relatively low, accounting for $4.6 \%$ of the total cost. A series of sensitivity analysis regarding the coefficients $r, \lambda_{B}, \beta$ and $\alpha$ are carried out to explore their influence on the optimisation result.

The coefficient $r$ stands for the charge rate of proof-ofwork in $€ / \mathrm{kWh}$, an important and representative by-product of the blockchain technology. In the base case above we set $r=0.01 € / \mathrm{kWh}$, which, obviously, indicates a relatively low level of the charge rate. Fig. 7 shows the trend of the total cost when $r$ varies from $0.01 € / \mathrm{kWh}$ to $100 € / \mathrm{kWh}$. As the cost of proof-of-work directly relates to the amount of electricity bought from the DA, adjustment and balancing markets, but has no relation with the contract, the ratio of electricity bought from contract increases with $r$ to avoid the high cost of proof-of-work. When $r=100$, almost $100 \%$ of the electricity are bought from the contract. The blue dotted line shows the total cost when $100 \%$ electricity is bought from the contract. Apparently, with $r$ increasing, the total cost gradually approaches the cost under $100 \%$ contract.

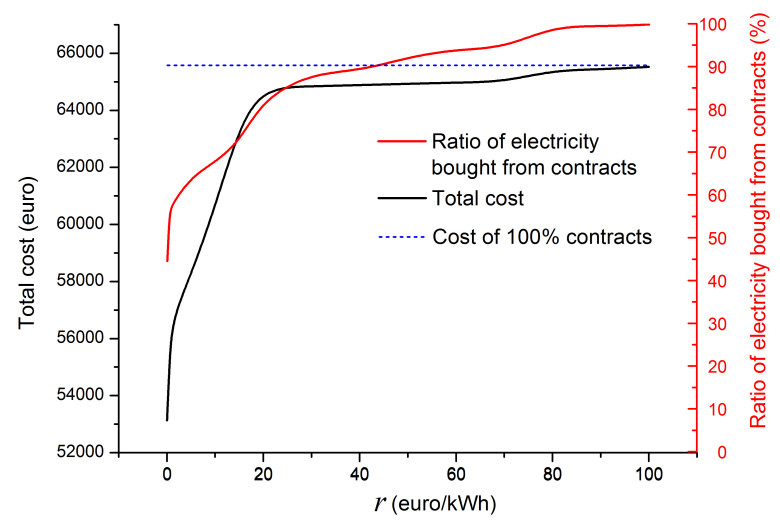

Fig. 7. Impact of proof-of-work charge rate $r$ on the total cost

The coefficient $\lambda_{B}$ refers to the penalty price for imbalance. Fig. 8 shows the relation between the value of $\lambda_{B}$, the total cost and the ratio of imbalance, i.e., the ratio of electricity bought from plus sold to the balancing market, in which $\lambda_{B}$ ranges from 0 to $50 € / \mathrm{kWh}$. Apparently, when $\lambda_{B}$ is high, the amount of electricity bought from plus sold to the balancing market decreases to nearly 0 . When the value of $\lambda_{B}$ is between 0 and $10 € / \mathrm{kWh}$, the total cost roughly increases linearly with respect to $\lambda_{B}$ with a slope of 2 . In reality, electricity cost can never be higher than $10 € / \mathrm{kWh}$; therefore, the total cost can be approximated by this linear segment with respect to $\lambda_{B} \in(0,10)$. Fig. 9 shows the relation between the risk

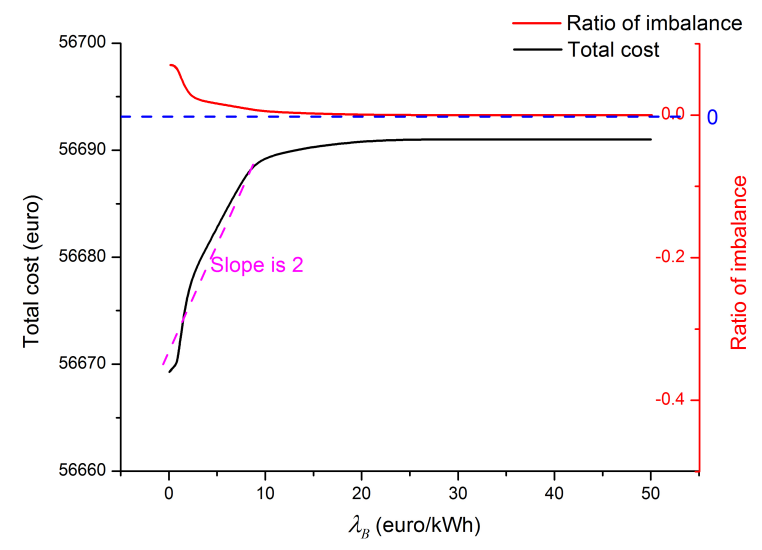

Fig. 8. Impact of imbalance penalty price $\lambda_{B}$ on the total cost

preference $\beta$ and the total cost. In simple words, the coefficient 
$\beta$ refers to the acceptability of risk, i.e., a higher $\beta$ means a risk is more likely to be accepted. As shown in Fig. 9, the total cost decreases when $\beta$ increases, as a higher beta renders less money to be spent to face the possible risk.

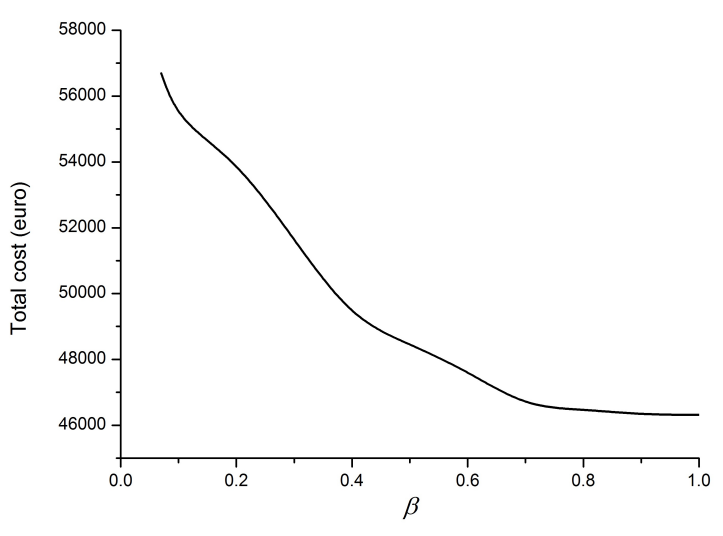

Fig. 9. Impact of risk preference $\beta$ on the total cost

Fig. 10 shows the variation of the total cost when the risk probability $\alpha$ ranges from 0 to 1 . The five different curves show how the total cost changes against $\alpha$ for five different combinations of $r, \lambda_{B}$ and $\beta$. The red line in the middle of the figure corresponds to the base case of $r=0.01, \lambda_{B}=0.05$ and $\beta=0.07$, and it shows that the total cost changes very little for different $\alpha$ 's. From the definition of CVaR in [34], the value of $C V a R_{\alpha}$ in (5) decreases with the increase of $\alpha$. Therefore, the total cost, i.e., the value of objective function in (8) decreases when $\alpha$ increases, which is also illustrated in Fig. 10. Although the five curves varies significantly regarding the total cost, they show the same decreasing trend with the increasing $\alpha$.

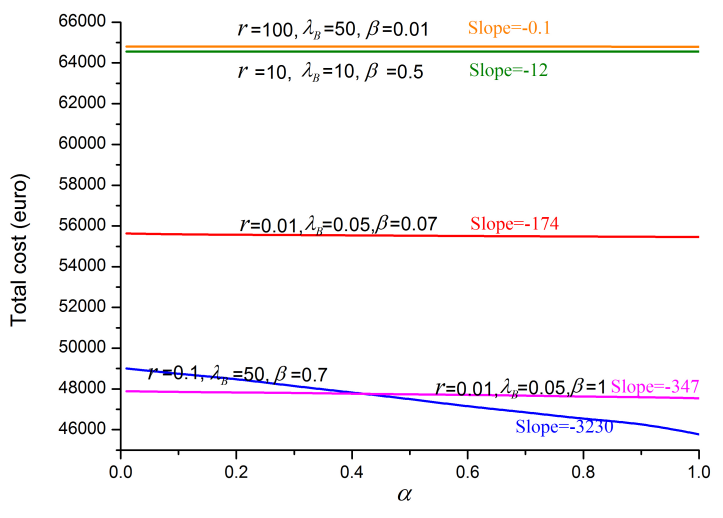

Fig. 10. Impact of risk probability $\alpha$ on the total cost

\section{B. Comparison with the spot market}

To compare the efficiency of the proposed P2P market structure, we compare the optimisation results with the same energy consumption scenario in the spot market, where the
TABLE II

Comparison between Case A and Case B

\begin{tabular}{lll}
\hline \hline & Case A & Case B \\
\hline Amount of electricity(kWh): & 687596 & 687596 \\
\hline Cost of buying electricity from $(€):$ & 31067 & 64807 \\
Contract & 13303 & 0 \\
DA market & 6259 & 0 \\
Adjustment market & 17 & 0 \\
Balancing market & 36 & 0 \\
\hline Income of selling electricity to Balancing market $(€)$ & 35 & 0 \\
\hline Cost of proof-of-work $(€)$ & 0.26 & 0 \\
\hline Penalty of unbalance $(€)$ & 50645 & 64839 \\
\hline Cost of buying \& selling electricity $(€)$ & 2491 & 723 \\
\hline Cost of risk $(€)$ & 53136 & 65530 \\
\hline Total cost $(€)$ & & \\
\hline \hline
\end{tabular}

latter is denoted as Case B. Noting that for most of the industrial users, the relevant spot market price is indeed a contracted time-of-use tariff, therefore, we use the contract price of Case A as the spot market price of Case B, which will also enable a fair cost comparison between Cases A and B.

The amount of electricity bought in Case B is $687596 \mathrm{kWh}$, which equals that of Case A. The cost of buying electricity in Case B is $€ 64807$, and the cost of risk is 723 . The total cost of Case B is $€ 65530,23.3 \%$ higher than Case A, or in other words, the optimal solution under P2P in Case A saves 18.9\% of the total cost compared with Case B. Detailed comparison between Case A and Case B is also provided in Table II. This difference shows that the load management model significantly saves the cost of buying electricity for big consumers under the proposed P2P market.

As discussed above, Case A has the optimised proportion of electricity bought from the contract, DA, adjustment and balancing markets, while Case $\mathrm{B}$ is equivalent to the case when $100 \%$ of the electricity comes from the contract. To further explore the relationship between the total cost and the proportion of the electricity bought from contract, we carry out a series of sensitivity analysis regarding different proportions of the amount of electricity bought from contract. In each case, we set an additional constraint on the amount of electricity bought from contract, then run the optimisation programme. The results are shown in Fig. 11. It can be seen that the lowest cost lies in Case A, i.e., the case without any additional constraints on the amount of electricity bought from contract. Case B has the highest cost, indicating an unitary market structure could be costly.

\section{Special cases under network constraint or self generation}

Now consider the proposed model under two special cases. The first case, named Case C, is to consider the power security constraint (18) for network security reasons, while the second case, named Case D, is to assume that the water supply plant has self PV generations. In Case $\mathrm{C}$, the total power supplied to the water supply plant is constrained by the network. As a result, this water supply plant has to adjust its load profile while satisfying the water demand. According to Case A, the load profile has a peak of $45.0 \mathrm{MW}$ between 15 o'clock and 17 o'clock that coincides with the peak load of the power 


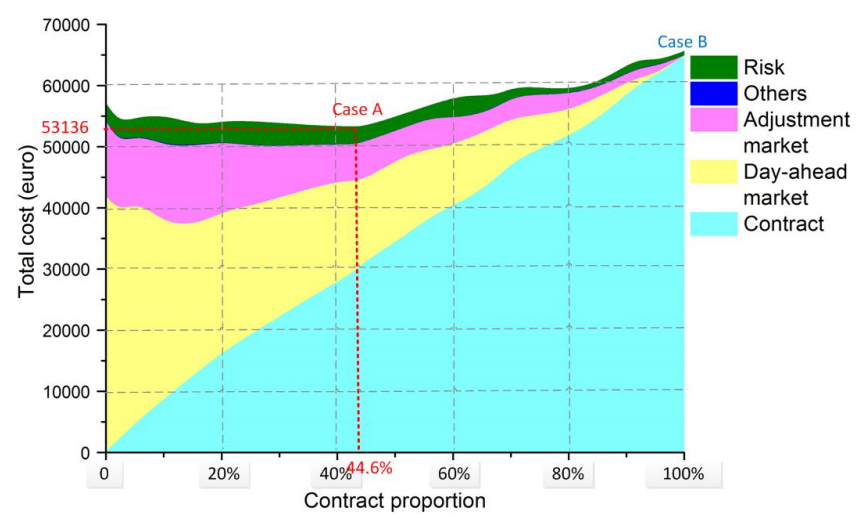

Fig. 11. Cost under different contract proportion

system. Therefore, in Case C, we consider a $80 \%$ restriction on the amount of power between 15 o'clock and 17 o'clock, i.e., a $20 \%$ power curtailment between 15 o'clock and 17 o'clock compared with the original load of Case A. The optimisation results are listed in Table III. Under the network constraint between 15 o'clock and 17 o'clock, $20 \%$ of the energy consumption of this period has to be shifted to other periods. Since Case A has the optimised energy transaction scheme, this shift of energy consumption in Case C implies certain amount of the energy transactions are shifted from lower price markets to higher price markets. As a result, the total cost of Case C is $0.28 \%$ higher than Case A. Table IV shows the increasing trend of total cost when the proportion of power curtailment increases. For example, when the power curtailment between 15 o'clock and 17 o'clock is $10 \%$ of the original power purchased under Case A, the total cost is $€ 53210$, which is $0.14 \%$ higher than Case A. When this power curtailment is as high as $50 \%$, then the total cost is $€ 53624$, which is only $0.92 \%$ higher than Case A and is still $18.2 \%$ lower than Case B when Case B is not subject to any network constraint. This shows that network constraint has only a very minor impact on the cost of the water supply plant. The main reason is that the network constraint is applied only during the peak hours 15-17 o'clock, and thus the water supply plant can take advantage of the reservoirs to store water and shift part of the load to other periods.

Since there are more and more renewable generations at the energy end user side, it is interesting to investigate the impact of renewable generation on the overall cost of the end users. In Case D, we assume that this water supply plant owns a 4 MW PV generation facility which supplies part of the power demand during the daytime. Comparison between Case A and Case D is also provided in Table III. It is observed that the amount of energy bought in Case D is $652903 \mathrm{kWh}$, while the PV system supplies $34693 \mathrm{kWh}$. The total cost of Case $\mathrm{D}$ is $4.4 \%$ lower than that of Case A, that is, the PV system can save $4.4 \%$ of the optimal cost in Case A or $22.5 \%$ of the unoptimised cost in Case B.
TABLE III

Comparison between Case A, CASE C and Case D

\begin{tabular}{llll}
\hline \hline & Case A & Case C & Case D \\
\hline Electricity bought from: & & & \\
Contract $(\mathrm{kWh})$ & 306775 & 341967 & 305555 \\
DA market $(\mathrm{kWh})$ & 247706 & 200822 & 192681 \\
Adjustment market $(\mathrm{kWh})$ & 133210 & 144842 & 154700 \\
Balancing market $(\mathrm{kWh})$ & 234 & 275 & 251 \\
\hline Electricity sold to & & & \\
Balancing market $(\mathrm{kWh})$ & 329 & 310 & 284 \\
\hline Total amount of transaction $(\mathrm{kWh}):$ & 687596 & 687596 & 652903 \\
\hline Cost of buying electricity from: & & & \\
Contract $(€)$ & 31067 & 33577 & 32680 \\
DA market $(€)$ & 13303 & 8642 & 7171 \\
Adjustment market $(€)$ & 6259 & 7800 & 9062 \\
Balancing market $(€)$ & 17 & 21 & 20 \\
\hline Income of selling electricity to: & & & \\
Balancing market $(€)$ & 36 & 26 & 34 \\
\hline Cost of proof-of-work $(€)$ & 35 & 29 & 26 \\
\hline Penalty of unbalance $(€)$ & 0.26 & 0.28 & 0.25 \\
\hline Cost of buying \& selling electricity $(€)$ & 50645 & 50043 & 48925 \\
\hline Cost of risk $(€)$ & 2491 & 3244 & 1873 \\
\hline Total cost $(€)$ & 53136 & 53287 & 50798 \\
\hline \hline
\end{tabular}

TABLE IV

SENSITIVITY ANALYSIS ABOUT THE NETWORK CONSTRAINT

\begin{tabular}{ll}
\hline \hline $\begin{array}{l}\text { Network constraint (proportion of power } \\
\text { curtailment to the original load) }\end{array}$ & Total Cost $(€)$ \\
\hline 0 & 53136 \\
\hline $10 \%$ & 53210 \\
\hline $20 \%$ & 53287 \\
\hline $30 \%$ & 53362 \\
\hline $40 \%$ & 53505 \\
\hline $50 \%$ & 53624 \\
\hline \hline
\end{tabular}

\section{CONClusion}

This paper proposes a new market structure, which can be implemented by any existing blockchain-based peer-to-peer electricity market platforms, and then investigates the optimal demand load management problem for a big industrial user. Case studies on a water supply plant show that the optimised load management under this new market structure significantly saves the electricity cost compared with the spot market, and network constraints at peak hours have only minor impact, i.e., less than $1 \%$, on the total electricity cost. The proposed peerto-peer market structure and load management models can also be applied in energy trading within a power distribution system, where the big consumer can be understood as a broker or an aggregator which integrates small loads. Since this paper studies only the situation of one big consumers, scenarios for multiple consumers will be studied in the future work.

Acknowledgement The authors would like to thank Dr Pedro Balaguer Herrero from the Jaume I University and $\mathrm{Mr}$ Sahand Ghavidel Jirsaraie from the University of Technology Sydney for their kind help. They are also grateful to the editor and anonymous reviewers for their insight and valuable comments which significantly improved the quality of the paper.

\section{REFERENCES}

[1] S Nakamoto, Bitcoin: A Peer-to-Peer Electronic Cash System, available at https://bitcoin.org/bitcoin.pdf (accessed on 1st September 2018). 
[2] G Chapron, The environment needs cryptogovernance, Nature, vol. 545, pp. 403-405, 2007.

[3] FutureThinkers, 19 Industries the Blockchain will Disrupt, available at https://www.youtube.com/watch? $\mathrm{v}=\mathrm{G} 3$ psxs3gyf8 or https://futurethinkers.org/industries-blockchain-disrupt/ (accessed on 1st September 2018).

[4] Australian Energy Market Commission, Distribution Market Model, Final Report, 2017.

[5] MJ Ashley and MS Johnson, Establishing a secure, transparent, and autonomous blockchain of custody for renewable energy credits and carbon credits, IEEE Engineering Management Review, DOI 10.1109/EMR.2018.2874967, to appear.

[6] G Liang, SR Weller, F Luo, J Zhang and ZY Dong, Distributed blockchain-based data protection framework for modern power systems against cyber attacks, IEEE Transactions on Smart Grid, DOI 10.1109/TSG.2018.2819663, to appear.

[7] E Mengelkamp, et al.,Designing microgrid energy markets: A case study: The Brooklyn Microgrid, Applied Energy, Vol. 210, Pages 870880.

[8] M Andoni, et al., Blockchain technology in the energy sector: A systematic review of challenges and opportunities, Renewable and Sustainable Energy Reviews, vol. 100, 2019, pp. 143-174.

[9] The GridWise Architecture Council, Gridwise Transactive Energy Framework, Version 1.0, Jan 2015, available: https://www.gridwiseac.org/pdfs/te_framework_report_pnnl-22946.pdf, accessed on 01/20/2019.

[10] JJ Sikorski, J Haughton, M Kraft, Blockchain technology in the chemical industry: Machine-to-machine electricity market, vol. 195, 2017, pp. 234-246.

[11] F Luo, ZY Dong, G Liang, J Murata and Z Xu, A distributed electricity trading system in active distribution networks based on multi-agent coalition and blockchain, IEEE Transactions on Power Systems, DOI 10.1109/TPWRS.2018.2876612, to appear.

[12] J Kang, et al., Enabling localized peer-to-peer electricity trading among plug-in hybrid electric vehicles using consortium blockchains, IEEE Transactions on Industrial Informatics, vol. 13, 2017, pp 3154-3164.

[13] Z Su, Y Wang, Q Xu, M Fei, YC Tian and N Zhang, A secure charging scheme for electric vehicles with smart communities in energy blockchain, DOI 10.1109/JIOT.2018.2869297, IEEE Internet of Things Journal, to appear

[14] $\mathrm{Z} \mathrm{Li}$, et al., Consortium blockchain for secure energy trading in industrial internet of things, IEEE Transactions on Industrial Informatics, vol. 14 2018, pp. 3690-3700.

[15] NZ Aitzhan and D Svetinovic, Security and privacy in decentralized energy trading through multi-signatures, blockchain and anonymous messaging streams, IEEE Transactions on Defendable and Secure Computing, vol. $15,2018,840-852$

[16] A Paudel, K Chaudhari, C Long, and HB Gooi, Peer-to-peer energy trading in a prosumer based community microgrid: A game-theoretic model, DOI 10.1109/TIE.2018.2874578, IEEE Transactions on Industrial Electronics, to appear.

[17] MR Alam, M St-Hilaire, and T Kunz, An optimal p2p energy trading model for smart homes in the smart grid, Energy Efficiency, vol. 10, pp. 1475-1493, 2017

[18] C Zhang, J Wu, Y Zhou, M Cheng and C Long, Peer-to-peer energy trading in a microgrid, Applied Energy, vol. 220, 2018, pp. 1-12.

[19] T Morstyn, A Teytelboym and MD McCulloch, Bilateral contract networks for peer-to-peer energy trading, IEEE Transactions on Smart Grid, DOI 10.1109/TSG.2017.2786668, to appear.

[20] $\mathrm{T}$ Chen and $\mathrm{W} \mathrm{Su}$, Indirect customer-tocustomer energy trading with reinforcement learning, IEEE Transactions on Smart Grid, DOI 10.1109/TSG.2018.2857449, to appear.

[21] R. Alvaro-Hermana, J Fraile-Ardanuy, PJ Zufiria, L Knapen and D Janssens, Peer to peer energy trading with electric vehicles, IEEE Intelligent Transportation Systems Magazine, Fall 2016, pp. 33-44.

[22] W Tushar, C Yuen, H Mohsenian-Rad, T Saha, HV Poor and KL Wood, Transforming energy networks via peer-to-peer energy trading, IEEE Signal Processing Magazine, July 2018, pp. 90-111.

[23] W Tushar, TK Saha, C Yuen, P Liddell, R Bean and HV Poor, Peer-topeer energy trading with sustainable user praticipation: A game theoretic approach, IEEE Access, vol.6, pp. 62932-62943.

[24] J Li, et al., Distributed transactive energy trading framework in distribution networks, IEEE Transactions on Power Systems, vol. 33, 2018, pp. 7215-7227.

[25] J Guerrero, AC Chapman and G Verbic, Decentralized P2P energy trading under network constraints in a low-voltage network, IEEE Transactions on Smart Grid, DOI 10.1109/TSG.2018.2878445, to appear.
[26] MLD Silvestre, A technical approach to the energy blockchain in microgrids, IEEE Transactions on Industrial Informatics, vol. 14, 2018 , pp. 4792-4803.

[27] YK Renani, M Ehsan and M Shahidehpour, Optimal transactive market operations with distribution system operators, IEEE Transactions on Smart Grid, vol. 9, 2018, pp. 6692-7601.

[28] T Morstyn and MD McCulloch, Multi-class energy managemen$\mathrm{t}$ for peer-to-peer energy trading driven by prosumer preferences, IEEE Transactions on Power Systems, to appear, DOI 10.1109/TPWRS.2018.2834472.

[29] AJ Conejo, M Carrion and JM Morales, Decision Making under Uncertainty in Electricity Markets, Springer, New York, 2010.

[30] JM Morales, AJ Conejo and J Perez-Ruiz, Short-term trading for a wind power producer, IEEE Transactions on Power Systems, vol. 25, 2010, pp. 554-564.

[31] N Liu, et al., Energy-sharing model with price-based demand response for microgrids of peer-to-peer prosumers, IEEE Transactions on Power Systems, vol. 32, 2017, pp. 3569-3583.

[32] J Aghaei, M Barani, M Shafie-khah, AA Sanchez de la Nieta and JPS Catalao, Risk-constrained offering strategy for aggregated hybrid power plant including wind power producer and demand response provider, IEEE Transactions on Sustainable Energy, vol.7, 2016, pp. 513-523.

[33] M Song and M Amelin, Price-maker bidding in day-ahead electricity market for a retailer with flexible demands, IEEE Transactions on Power Systems, to appear, DOI 10.1109/TPWRS.2017.2741000

[34] RT Rockafellar and S Uryasev, Optimization of conditional value-at-risk, Journal of Risk, vol.2, 2000, pp. 21-41.

[35] A Shapiro, D Dentcheva and A Ruszczynski, Lectures on Stochastic Programming-Modeling and Theory, 2nd Edition, SIAM, Philadelphia, 2014.

[36] J Zhang, X Xia and D Alexander, Demand side optimal strategy for voluntary load shedding, The 2nd IASTED Africa Conference on Power and Energy System (AfricaPES 2008), 8-10 September, 2008, Gaborone, Botswana.

[37] S Ghavidel Jirsaraie, M Barani, A Azizivahed, L Li and J Zhang, Hybrid power plant offering strategy to deal with the stochastic nature and outage of wind generators, 20th International Conference on Electrical Machines and Systems (ICEMS), Sydney, 11-14 Aug 2017. 